\title{
Short communication: Molecular typing of Prototheca zopfii from bovine mastitis in Japan
}

\author{
H. Sobukawa, ${ }^{*}$ S. Yamaguchi, ${ }^{*}$ R. Kano, ${ }^{* 1}$ T. Ito,† K. Suzuki, ¥ M. Onozaki,§ A. Hasegawa,\# and H. Kamata* \\ *Department of Pathobiology, Nihon University School of Veterinary Medicine, 1866 Kameino, Fujisawa, Kanagawa 252-8510, Japan \\ †Okazaki Branch of Veterinary Hospital Aichi P.F.A.M.A.A., 91-1, Oike, Hane-cho, Okazaki, Aichi 444-0816, Japan \\ łDepartment of Large Animal Clinical Sciences, School of Veterinary Medicine, Rakuno Gakuen University, 582 Midorimati, Bunnkyoudai, \\ Ebetsu, Hokkaido 069-8501, Japan \\ §Kanto Chemical Co. Inc., Marusan Building 11-5, Nihonbashi Honcho, 3-Chome, Chuo-ku, Tokyo 103-0023, Japan \\ \#Teikyo University Institute of Medical Mycology, 539 Otsuka, Hachioji, Tokyo 192-0395, Japan
}

\begin{abstract}
Prototheca zopfii causes bovine mastitis, resulting in reduced milk production and the secretion of thin watery milk with white flakes. Prototheca zopfii has been biochemically and serologically divided into at least 2 genotypes, P. zopfii genotype 1 and P. zopfii genotype 2. The latter is known to be the main causative agent of bovine protothecal mastitis. Prototheca zopfii was later reclassified into 5 varieties: var. zopfii (genotypes 1 and 2), var. 1 (formerly Prototheca blaschkeae), var. 3 (formerly P. moriformis), and var. portoricensis. In this study, the $18 \mathrm{~S}$ ribosomal DNA sequences of diverse clinical specimens from different areas in Japan were studied to clarify the pathogenicity of $P$. zopfii var. zopfii. The phylogenetic tree revealed that all genotype 2 isolates were grouped in a cluster of $P$. zopfii var. zopfii SAG $2021^{\mathrm{T}}$ (type strain genotype 2), and were independent from the cluster of the genotype 1 isolates. Thus, all isolates from bovine mastitis in Japan were identified as $P$. zopfii genotype 2. Therefore, P. zopfii var. zopfii genotype 2 is associated with bovine mastitis. Key words: bovine Prototheca mastitis, genotype, Prototheca zopfii, $18 \mathrm{~S}$ ribosomal DNA
\end{abstract}

\section{Short Communication}

The genus Prototheca consists of achlorophyllic algae that are ubiquitous among cow-barn surroundings. Prototheca zopfii is associated with bovine mastitis, which causes a reduction in milk production and the secretion of thin, watery milk with white flakes. Prototheca zopfii has been biochemically and serologically divided into at least 2 genotypes, $P$. zopfii genotype 1 and $P$. zopfii

Received October 28, 2011.

Accepted April 11, 2012.

${ }^{1}$ Corresponding author: kano@brs.nihon-u.ac.jp genotype 2 (Roesler et al., 2003, 2006). Isolates from bovine mastitis in Germany, Italy, Japan, Portugal, and Poland were almost all identified as $P$. zopfii genotype 2 , suggesting that it is the main causative agent of bovine mastitis (Roesler et al., 2003, 2006; Möller et al., 2007; Marques et al., 2008; Osumi et al., 2008; Ricchi et al., 2010; Jagielski et al., 2011). Afterward, Pore (2011) reclassified P. zopfii into 5 varieties: var. zopfii (genotypes 1 and 2), var. 1 (formerly Prototheca blaschkeae), var. 3 (formerly P. moriformis), and var. portoricensis. However, further studies on the molecular typing of P. zopfii var. zopfii (genotypes 1 and 2) are needed to ascertain its genetic characteristics as well as its etiologic role in bovine mastitis. In this study, the $18 \mathrm{~S}$ ribosomal ( $\mathbf{r}) \mathrm{DNA}$ sequences of diverse clinical specimens from different areas in Japan were studied to clarify the pathogenicity of $P$. zopfii var. zopfii.

The $P$. zopfii type strains of $P$. zopfii var. 1 $\left(\mathrm{SAG} 2064^{\mathrm{T}}\right)$, genotype $1\left(\mathrm{SAG} 2063^{\mathrm{T}}\right)$, and genotype 2 $\left(\mathrm{SAG} 2021^{\mathrm{T}}\right.$ ) were used for this study. In addition to the type strains, 1 isolate of $P$. zopfii var. 1 and 21 clinical strains of P. zopfii were isolated from 21 cases of bovine (Holstein cow) mastitis from the Aichi, Chiba, and Hokkaido areas in Japan (Table 1). One strain of P. zopfii var. 1 and 10 strains of genotype 1 were isolated from farm stock in the Aichi area (Table 1), as described in our previous study (Osumi et al., 2008; Kishimoto et al., 2010).

All strains were grown on Sabouraud dextrose agar at $27^{\circ} \mathrm{C}$ for $1 \mathrm{wk}$, and were then collected by scraping and suspended at $37^{\circ} \mathrm{C}$ for $14 \mathrm{~h}$ in lysis buffer [1 mg/ $\mathrm{mL}$ of zymolyase 100T (Takara, Kyoto, Japan), $0.1 \mathrm{mM}$ EDTA, 1\% SDS, $10 \mathrm{~m} M$ Tris hydrochloride ( $\mathrm{pH} 8.0$ ), and $0.3 \%$ 2-mercaptoethanol].

The primer set for the protothecal $18 \mathrm{~S}$ rDNA domain was designed by Roesler et al. (2006) as follows: Proto 18S-4F (5'-GACATGGCGAGGATTGACAGA-3') and Proto 18S-4R (5'-AGCACACCCAATCGGTAGGA-3'), where $\mathrm{F}$ is forward and $\mathrm{R}$ is reverse. 
Table 1. Prototheca isolates in Japan used in this study

\begin{tabular}{|c|c|c|c|}
\hline Variety & Strain & Origin & Genotype $^{1}$ \\
\hline var. 1 & $\mathrm{O} 2$ & Bovine feces & \\
\hline var. zopfii & NUBS 1 & Bovine mastitis in Aichi & 2 \\
\hline var. zopfii & NUBS 24 & Bovine mastitis in Aichi & 2 \\
\hline var. zopfii & NUBS 26 & Bovine mastitis in Aichi & 2 \\
\hline var. zopfii & NUBS 28 & Bovine mastitis in Aichi & 2 \\
\hline var. zopfii & NUBS 44 & Bovine mastitis in Aichi & 2 \\
\hline var. zopfii & NUBS 69 & Bovine mastitis in Aichi & 2 \\
\hline var. zopfii & NUBS 89 & Bovine mastitis in Aichi & 2 \\
\hline var. zopfii & NUBS 101 & Bovine mastitis in Aichi & 2 \\
\hline var. zopfii & NUBS 114 & Bovine mastitis in Aichi & 2 \\
\hline var. zopfii & NUBS 118 & Bovine mastitis in Aichi & 2 \\
\hline var. zopfii & $\mathrm{T} 1$ & Bovine mastitis in Chiba & 2 \\
\hline var. zopfii & $\mathrm{T} 2$ & Bovine mastitis in Chiba & 2 \\
\hline var. zopfii & T3 & Bovine mastitis in Chiba & 2 \\
\hline var. zopfii & $\mathrm{T} 4$ & Bovine mastitis in Chiba & 2 \\
\hline var. zopfii & T5 & Bovine mastitis in Chiba & 2 \\
\hline var. zopfii & T6 & Bovine mastitis in Chiba & 2 \\
\hline var. zopfii & $\mathrm{T} 7$ & Bovine mastitis in Chiba & 2 \\
\hline var. zopfii & $\mathrm{T} 8$ & Bovine mastitis in Chiba & 2 \\
\hline var. zopfii & B397 & Bovine mastitis in Hokkaido & 2 \\
\hline var. zopfii & D397 & Bovine mastitis in Hokkaido & 2 \\
\hline var. zopfii & D455 & Bovine mastitis in Hokkaido & 2 \\
\hline var. zopfii & NUBS 73 & Sewage & 1 \\
\hline var. zopfii & NUBS 65 & Bovine feces & 1 \\
\hline var. zopfii & NUBS 68 & Bovine feces & 1 \\
\hline var. zopfii & NUBS 74 & Bovine feces & 1 \\
\hline var. zopfii & NUBS 82 & Bovine feces & 1 \\
\hline var. zopfii & NUBS 88 & Bovine feces & 1 \\
\hline var. zopfii & NUBS 21 & Drinking water & 1 \\
\hline var. zopfii & NUBS 41 & Drinking water & 1 \\
\hline var. zopfii & NUBS 70 & Drinking water & 1 \\
\hline var. zopfii & NUBS 117 & Drinking water & 1 \\
\hline
\end{tabular}

${ }^{1}$ Identified by $18 \mathrm{~S}$ ribosomal DNA sequence analysis.

Thirty-five cycles of PCR amplification were performed under the following conditions: denaturation for $30 \mathrm{~s}$ at $95^{\circ} \mathrm{C}$, primer annealing for $1 \mathrm{~min}$ at $58^{\circ} \mathrm{C}$, and polymerization for $1 \mathrm{~min}$ at $72^{\circ} \mathrm{C}$ in a total reaction volume of $20 \mu \mathrm{L}$ of amplification mixture [10 $\mathrm{m} M$ Tris- $\mathrm{HCl}(\mathrm{pH} 8.3), 50 \mathrm{~m} M \mathrm{KCl}, 1.5 \mathrm{mM} \mathrm{MgCl}{ }_{2}$, $0.001 \%$ gelatin, $200 \mathrm{~m} M$ deoxynucleotide triphosphate, $1.0 \mathrm{U}$ of $\mathrm{Taq}$ polymerase (Takara), and $0.5 \mu \mathrm{g}$ of each primer]. The resultant amplified DNA fragments were electrophoresed on a $2 \%(\mathrm{wt} / \mathrm{vol})$ agarose gel with $1 \times$ Tris-acetate-EDTA buffer and visualized by ethidium bromide.

A DNA band of approximately 450 bp for each strain was excised from the gel and then purified with an ExoSAP-IT kit (USB Corporation, Cleveland, $\mathrm{OH}$ ) and sequenced on an ABI PRISM 310 Genetic Analyzer (Applied Biosystems, Foster City, CA).

The resulting sequences were compared with those of the type strains and isolates (Table 1) by using the ClustalW multiple-sequence alignment program (Thompson et al., 1994). The phylogenetic tree was constructed by the neighbor-joining method using the TreeView program (Page, 1996). Bootstrap analysis was performed on 100 random samples and analyzed by the ClustalW program (Felsenstein, 1985).

This study was performed to clarify the phylogenetic relationships among the genotypes of $P$. zopfii var. zopfii and other varieties of $P$. zopfii. The phylogenetic analysis of clinical isolates based on the $18 \mathrm{~S} \mathrm{rDNA}$ sequences revealed that these isolates were grouped in a cluster of $P$. zopfii var. zopfii SAG $2021^{\mathrm{T}}$ (type strain genotype 2) and P. zopfii var. 3 ATCC 50081 (GenBank accession number AB077048), and were independent from the cluster of the genotype 1 isolates (Figure 1). Prototheca zopfii var. 3 is differentiated from var. zopfii by capsule production and has been regarded as nonpathogenic (Pore, 2011). In this study, we sequenced a type strain $\left(\mathrm{SAG} 2064^{\mathrm{T}}\right.$ ) and 1 clinical isolate of $P$. zopfii var. 1 (formerly P. blaschkeae). The phylogenetic analysis of $18 \mathrm{~S}$ rDNA sequences also indicated that $P$. $z o p f i i$ var. 1 was genetically distinct from the other $P$. zopfii varieties. Therefore, $P$. zopfii var. 1 should be classified as a different species (formerly P. blaschkeae) from P. zopfii.

The resulting sequences were also aligned, and the phylogenetic tree was constructed by maximum par- 


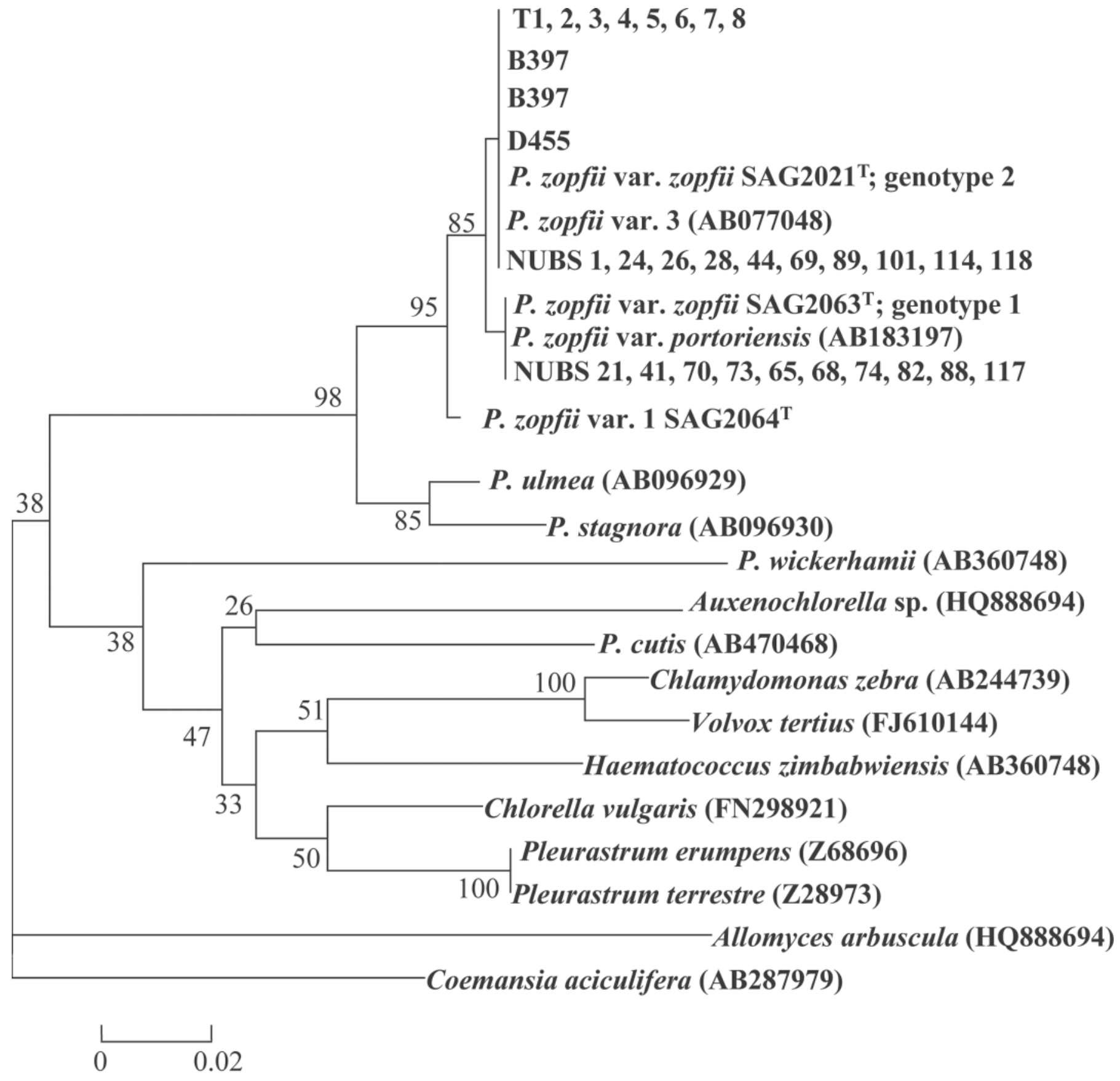

Nucleotide substitute/site

Figure 1. A phylogenetic tree by the neighbor-joining method showing the molecular taxonomy of $18 \mathrm{~S}$ ribosomal gene fragments. The numbers at the branches were determined by the bootstrap value indicating the times in 100 repeat subsamples in a monophylogenic grouping. Items in parentheses indicate the GenBank accession number of the $18 \mathrm{~S}$ ribosomal gene of algae species.

simony using the program MEGA 5.1 (http://www. megasoftware.net/). The phylogenetic tree created by maximum parsimony also indicated the neighbor-joining tree constructed from the current study (Figure 2).
Recently, genotypes 1 and 2 were reclassified as the same variety of P. zopfii (Pore, 2011). In our previous report, we examined the susceptibility of $P$. zopfii genotype 1 and 2 isolates to amphotericin $\mathrm{B}$, gentamicin, 


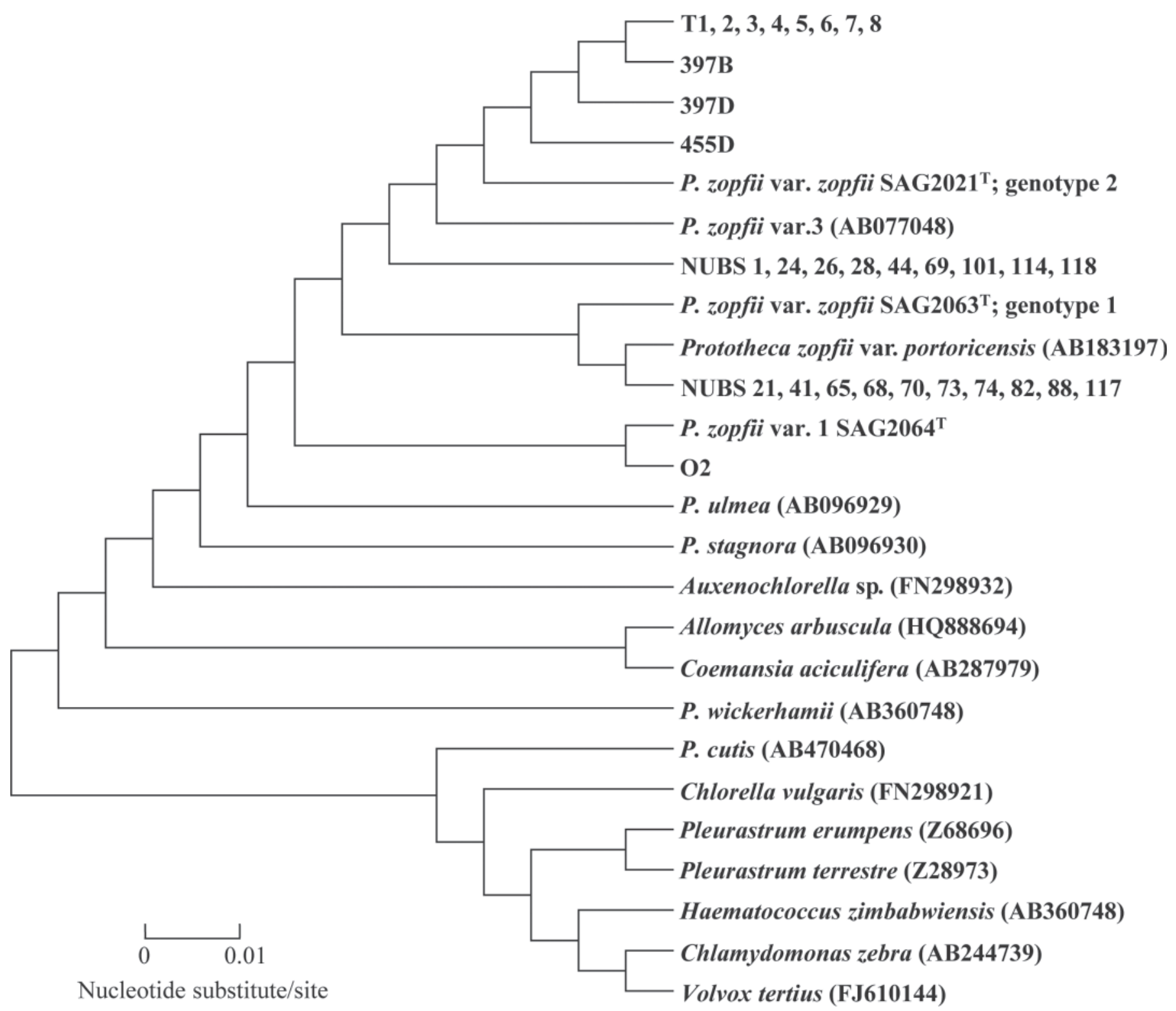

Figure 2. A phylogenetic tree by the maximum parsimony method showing the molecular taxonomy of $18 \mathrm{~S}$ ribosomal gene fragments. Items in parentheses indicate the GenBank accession number of the $18 \mathrm{~S}$ ribosomal gene of algae species.

kanamycin, and itraconazole by E-test (Sobukawa et al., 2011). Prototheca zopfii var. zopfii genotype 1 was more susceptible to amphotericin B, gentamicin, and kanamycin than was $P$. zopfii var. zopfii genotype 2 (Sobukawa et al., 2011). These 2 genotypes are distinct in phenotype, pathogenicity, and $18 \mathrm{~S}$ rDNA sequence. Therefore, $P$. zopfii var. zopfii remains to be reclassified at the variety level. The reservoir of the etiologic agent of bovine mastitis is thought to be the environment because $P$. zopfii is often found in flowing and standing water. However, these environmental isolates belong to genotype 1 , whereas clinical isolates from bovine mastitis were identified as genotype 2. Further molecular typing of P. zopfii var. zopfii (genotypes 1 and 2) needs to be completed to identify preventative measures for bovine mastitis.

\section{ACKNOWLEDGMENTS}

This study was supported by Grants-in-Aid for Scientific Research (C; no. 22580368) from the Academic Frontier Project of the Japanese Ministry of Education, 
Culture, Sports, Science and Technology (MEXT, Tokyo, Japan), and from Nihon University. The authors report no conflicts of interest. The authors alone are responsible for the content and writing of the paper.

\section{REFERENCES}

Felsenstein, J. 1985. Confidence limits on phylogenies: An approach using the bootstrap. Evolution 39:783-791.

Jagielski, T., H. Lassa, J. Ahrholdt, E. Malinowski, and U. Roesler. 2011. Genotyping of bovine Prototheca mastitis isolates from Poland. Vet. Microbiol. 149:283-287.

Kishimoto, Y., R. Kano, H. Maruyama, M. Onozaki, K. Makimura, T. Ito, K. Matsubara, A. Hasegawa, and H. Kamata. 2010. 26S rDNA-based phylogenetic investigation of Japanese cattle-associated Prototheca zopfii isolates. J. Vet. Med. Sci. 72:123-126.

Marques, S., E. Silva, C. Kraft, J. Carvalheira, A. Videira, V. A. Huss, and G. Thompson. 2008. Bovine mastitis associated with Prototheca blaschkeae. J. Clin. Microbiol. 46:1941-1945.

Möller, A., U. Truyen, and U. Roesler. 2007. Prototheca zopfii genotype 2-The causative agent of bovine protothecal mastitis? Vet. Microbiol. 120:370-374.

Osumi, T., Y. Kishimoto, R. Kano, M. Maruyama, M. Onozaki, K. Makimura, T. Ito, K. Matsubara, and A. Hasegawa. 2008. Prototheca zopfii genotypes isolated from cow barns and bovine mastitis in Japan. Vet. Microbiol. 131:419-423.
Page, R. D. M. 1996. TREEVIEW: An application to display phylogenetic trees on personal computers. Comput. Appl. Biosci. $12: 357-358$

Pore, R. S. 2011, Prototheca Krüger (1984). Pages 2071-2080 in The Yeasts, a Taxonomic Study. Vol. 3. 5th ed. C. P. Kurtzman, J. W Fell, and T. Boekhout, ed. Elsevier, Amsterdam, the Netherlands.

Ricchi, M., M. Goretti, E. Branda, G. Cammi, C. A. Garbarino, B. Turchetti, P. Moroni, N. Arrigoni, and P. Buzzini. 2010. Molecular characterization of Prototheca strains isolated from Italian dairy herds. J. Dairy Sci. 93:4625-4631.

Roesler, U., A. Möller, A. Hensel, D. Baumann, and U. Truyen. 2006. Diversity within the current algal species Prototheca zopfii: A proposal for two Prototheca zopfii genotypes and description of a novel species, Prototheca blaschkeae sp. nov. Int. J. Syst. Evol. Microbiol. 56:1419-1425.

Roesler, U., H. Scholz, and A. Hensel. 2003. Emended phenotypic characterization of Prototheca zopfii: A proposal for three biotypes and standards for their identification. Int. J. Syst. Evol. Microbiol. 53:1195-1199.

Sobukawa, H., R. Kano, T. Ito, M. Onozaki, K. Makimura, A. Hasegawa, and H. Kamata. 2011. In vitro susceptibility of Prototheca zopfii genotypes 1 and 2. Med. Mycol. 49:222-224.

Thompson, J. D., D. G. Higgins, and T. J. Gibson. 1994. CLUSTAL $\mathrm{W}$ : Improving the sensitivity of progressive multiple sequence alignment through sequence weighting, position-specific gap penalties and weight matrix choice. Nucleic Acids Res. 22:4673-4680. 